\title{
Réflexions prospectives sur l'éducation permanente
}

\author{
par Bertrand SCHWARTZ *
}

\section{PRÉAMBULE}

Ce qui a guidé nos réflexions n'est pas une image a priori de l'éducation permanente qu'on essaierait ensuite de justifier et de traduire en un système raisonnable, c'est-à-dire immédiatement accessible par tous et réalisable dans son ensemble, dès à présent.

Plutôt que de nous en tenir à des idées, des événements probables, à partir desquels il faudrait nous adapter, nous avons préféré une démarche prospective attirant l'attention sur l'avenir de l'éducation permanente, en l'imaginant à partir de l'avenir et non à partir du présent. D'ailleurs, il n'y a pas de présent de l'éducation permanente.

Notre réflexion n'est pas rétrospective, c'est-à-dire spéculative, mais veut être une prospection, c'est-àdire une pensée orientée vers l'action. Cependant, nous ne voulons pas laisser ici l'illusion d'un projet élaboré pour un avenir de vingt ans. Nous n'avons pas l'intention de proposer un projet mais un objet de réflexion, point de départ de projets possibles et d'expériences souhaitables.

* L'auteur est conseiller a l'Education permanente auprès du ministre de l'Education nationale en France.
Une des a règles du jeu, en matière de prospective est de se donner le droit de tout réimaginer, de tout remettre en cause et de tout reconstruire. Ceci nous a amené à proposer un système global qui veut dépasser donc les réformes partielles et les simples mesures d'adaptation.

Peut-être certains y verront-ils, en se tenant aux détails, des choses existant déjà et jugeront du tout comme peu novateur; d'autres, réagissant à l'ensemble du système, le jugeront visionnaire.

Nous voulons encore attirer l'attention sur un point essentiel. Nous ne voulons porter aucun jugement critique sur le système éducatif, sauf dire qu'il n'est pas du tout orienté vers l'éducation permanente : il y a juxtaposition d'un système éducatif scolaire et d'un certain nombre d'expériences et de réalisations postscolaires.

\section{D'ABORD DEEFINIR LES OBJECTIFS}

Il ne peut $\mathrm{y}$ avoir éducation, sans que les objectifs de cette éducation aient été pleinement définis, et ce, depuis les niveaux les plus généraux (ceux du 
ministère de l'Éducation nationale) jusqu'aux niveaux les plus particuliers (celui de l'enseignant). Enseigner n'est pas un objectif en soi, plus exactement la finalité de cette activité contient trop de choses pour se résumer en un seul objectif. Mais \& apprendre $\gg$, ou se former, n'est pas non plus un objectif en soi, et les finalités ne peuvent pas non plus, ni être résumées en un objectif, ni être laissées sans définition, ni être acceptées sans discussion par ceux qui apprennent.

Pour être un truisme, ce principe n'en est pas moins, ayons le courage de le dire, trop souvent négligé.

Au risque d'être, en effet, sévèrement jugé, disons que:

- le ministère de l'Éducation nationale n'a jamais défini ses objectifs généraux: en tout cas, ni en tant qu'enseignant, ni en tant que responsable d'institutions éducatives, nous n'avons jamais connu, ni officiellement, ni officieusement, les objectifs fixés par le ministère de l'Éducation nationale;

- les enseignants, s'ils poursuivent le plus souvent des objectifs précis, ne les confrontent guère entre eux, et ne les explicitent, et en tout cas ne les discutent presque jamais avec leurs élèves.

Or, des objectifs dépendront à la fois les structures, les contenus et les méthodes. Ce point nous paraît si essentiel qu'avant toute autre réflexion, nous allons étudier le problème en le pensant successivement au niveau de ceux qui forment, puis au niveau de ceux qui se forment.

\section{Le ministère de l'Éducation nationale doit commencer}

Les objectifs possibles sont très nombreux et, par exemple, peuvent être de:

- former l'esprit ou le comportement, en vue de la vie professionnelle, ou civique, ou familiale;

- transmettre l'héritage culturel;

- former les jeunes seuls ou les jeunes et les adultes;

— s'adapter aux besoins de l'emploi.

Is peuvent être aussi n'importe quelle combinaison de ces différents points.
Et chacune des hypothèses qui précèdent recouvre, à son tour, un ensemble de sous-hypothèses. A titre d'exemple, former le comportement recouvre de nombreux possibles, même si l'on réduit ce thème à la formation du caractère. On peut vouloir former des hommes obéissants, automates, ou autonomes, disponibles, persévérants, individualistes ou aptes au travail en équipe. C'est donc tout le système de valeurs qui est à définir et c'est, en fait, un problème politique.

\section{Les institutions éducatives doivent, à leur tour, préciser leurs objectifs}

Partant des objectifs de l'Éducation nationale, chaque institution de formation doit, elle-même, se définir ses objectifs propres. Par exemple, si l'École normale est chargée de former des professeurs et une École de Mines de former des ingénieurs des Mines, ces deux institutions auront de nombreux objectifs différents. Mais, que met-on derrière ces termes de professeur ou d'ingénieur des Mines et quel type d'hommes veut-on former? Cela exige d'être précisé en une série d'objectifs qui, bien entendu, ne peuvent pas être en contradiction avec ceux de l'Éducation nationale. Une école d'ingénieurs, par exemple, ne doit pas former des hommes autonomes, si l'ensemble du système éducatif fait des hommes dépendants.

Or, une institution qui veut, par exemple, développer l'autonomie doit instaurer un système de relation maître-élèves très différent de celle qui n'aurait pas cet objectif: dans une ambiance d'autonomie, les examens traditionnels doivent être supprimés pour que les élèves (avec l'aide de leurs maîtres) apprennent à \& se situer ${ }^{1}$ » et les cours qui apportent du \& tout pensé » doivent laisser place à beaucoup de recherches personnelles. D'où toute une série de mesures à prendre.

Et il en est de même pour tout autre objectif. Prenons un autre exemple, celui des infirmières: veut-on en faire des personnes autonomes et jusqu'où? De quels actes médicaux seront-elles responsables ? Veut-on qu'elles soient immédiatement utilisables ou, au contraire, capables de s'adapter constamment aux changements ? Seront-elles spécialisées, restant toujours dans le même service ou polyvalentes et changeant souvent ?

Contenus et méthodes de formation ne seront définis qu'après que toutes les réponses à ces questions auront été données.

\footnotetext{
1. Voir plus loin le problème de e l'auto-évaluation s.
} 


\section{Puls c'est au tour des enseignants}

Chaque enseignant à son niveau doit, à l'intérieur du cadre global de l'institution de formation dans lequel il travaille, se fixer ses propres objectifs. Imaginons une école qui se serait donné comme objectif de développer l'initiative et l'autonomie; communiquer des connaissances afin qu'elles soient assimilées par les élèves est, certes, la première mission du professeur, mais il devra participer à l'action générale de développement de l'autonomie, et cela influence directement, nous venons de le voir, la nature de ses relations avec ses élèves et jusqu'au mode de transmission des connaissances; il pourra, en outre, à titre personnel, vouloir développer telle ou telle aptitude: par exemple, en rapport avec les outils de pensée dont il arme les élèves, s'il est professeur de sciences par exemple, il peut se préoccuper de développer l'esprit d'observation ou, s'il est professeur de lettres ou de dessin, s'attacher à affiner les moyens d'expression qu'il y a en propre dans chacun de ses élèves.

En disant ce qui précède, nous avons voulu souligner qu'en l'absence d'une réflexion constante et approfondie sur les objectifs et sur les moyens correspondants à mettre en œuvre, il est difficile de dépasser le seul objectif d'apport de connaissances organisées en programmes, et ce d'autant plus que le modèle culturel traditionnel nous $y$ pousse très fortement: n'avons-nous pas, en effet, comme objectif inconscient, de former nos élèves à notre image, des élèves conformes à la représentation traditionnelle de « l'homme cultivé \$?

Or, la notion de programme nous semble, en l'occurrence, sans signification et il est nécessaire de préciser ce que l'on veut faire. Les programmes varieront en effet selon l'objectif qu'on recherche. Pour éclairer ce point, nous donnerons ici quatre formes d'objectifs parce qu'elles nous paraissent avoir un caractère général, et nous les expliciterons tout de suite par des exemples, afin d'être concrets:

Comme premier exemple, nous prendrons celui d'une discipline scientifique: la statistique. L'enseignement peut se limiter:

d l'information, en montrant que la loi mathématique fonctionnelle existe peu dans la nature, que le phénomène aléatoire est un phénomène général, qu'il existe des méthodes statistiques d'analyse des problèmes;
- au * moyen d'expression statistique s: cela veut dire, pour nous, d'exercer les élèves à formuler leurs problèmes, à présenter leurs données de manière probabiliste et statistique, à comprendre les résultats d'une étude de spécialiste:

- a l'outil statistique, en apprenant à manier les techniques de calcul en statistiques, à dresser et mettre en cuvre les plans d'expériences, à en assurer le dépouillement, à chiffrer le degré d'incertitude d'un résultat;

- à la formation méthodologique, en initiant les élèves au mode d'approche des problèmes encombrés par des facteurs multiples, à l'usage de l'expérimentation, à la démarche qui consiste à tester une hypothèse, au cheminement par induction.

En appliquant un découpage semblable à l'enseignement des sciences naturelles, par exemple, on fait ressortir combien pourrait être transformé profondément l'enseignement de cette discipline au secondaire 2 . Vraisemblablement, on en viendrait à redistribuer de la façon suivante ce que les élèves ont à apprendre actuellement: une certaine partie serait définitivement supprimée, celle qu'un esprit normalement constitué ne peut retenir qu'une quinzaine de jours; une autre partie se limiterait au niveau de l'information, une troisième partie consisterait dans l'apprentissage du langage de base (expression), qui permet de définir et décrire les phénomènes et les êtres de cette discipline, une quatrième partie, l'outil, ne serait enseignée qu'à un nombre limité d'élèves et ferait l'objet d'options entre lesquelles les élèves se répartiraient: un domaine encore plus restreint serait le cadre optimum pour aborder, avec une fraction plus ou moins importante de l'effectif, l'apprentissage d'une méthodologie d'observation et le maniement des moyens de recherche.

Ceci illustre ce que nous entendons par la définition des objectifs.

\section{Mais la définition des objectifs n'est pas l'apanage des enseignants}

Nous avons déjà souligné le fait que, souvent, les objectifs des professeurs étaient inconnus des élèves, d'où l'idée de voir s'instaurer une discussion entre le professeur et les élèves à propos des objectifs. Cela ne signifie pas que les élèves doivent pouvoir refuser les objectifs du professeur, voire refuser tel ou tel enseignement, mais nous pensons tout simplement que

2. Notons, puisqu'il est pris comme exemple, qu'il commence à être l'objet de nettes améliorations. 
le fait, pour le professeur, d'expliquer en permanence aux élèves pourquoi il fait ce qu'il fait, est le premier acte éducatif; parce qu'amenant les élèves à être plus motivés et surtout à mieux comprendre. Celà passe non seulement par une discussion, mais même, éventuellement, par une négociation entre élèves et professeur: nous youlons dire par là que le professeur doit, au début de son enseignement, et, à la limite, au début de chaque série de cours, expliciter les objectifs qu'il va suivre, les discuter avec les élèves et, s'il leur impose éventuellement pendant quelque temps tel ou tel contenu et telle ou telle méthode, c'est à la condition qu'après une série de séances il évalue, avec ses élèves, le décalage qui existe entre objectifs et résultats, qu'ensemble ils apprécient les raisons qu'avait l'enseignement de choisir l'itinéraire qu'il avait imposé.

Il ne s'agit pas d'une critique « du monde ou de la société \$, mais - ce n'est pas moins inquiétant pour le professeur - des raisons qui l'avaient conduit à choisir, ainsi, ses objectifs et les moyens mis en œuvre pour les atteindre.

D'autre part, cela amène à l'idée des options: tous les éducateurs sont d'accord pour penser que tous les élèves ne sont pas obligés de suivre la même formation et que, grâce à un système d'options diversifié, les élèves choisissent des matières pour lesquelles ils se sentiront le plus motivés. Mais, grâce à cette procédure de discussion approfondie des objectifs, c'est en connaissance de cause que les élèves choisiront leurs options, et non, comme c'est très souvent le cas aujourd'hui, n'importe comment ou; pire encore, par \& élimination ».

\section{En termes de conclusion}

Nous avons vu la nécessité d'une définition des objectifs, d'une définition en chaîne, à laquelle participent en particulier ceux qui se forment, et qui détermine structures, contenus et méthodes.

\section{QU'ASSIGNONS-NOUS COMME OBJECTIFS AU SYSTEMME ÉDUCATIF?}

\section{Proposition d'une définition}

Nous proposerons, comme définition de l'éducation, la définition suivante, que nous pensons être en conformité avec la pensée exprimée par le ministre de l'Education nationale, M. Edgar Faure, dans la Loi d'orientation:

Rendre capable toute personne de mieux comprendre le monde technique, social et culturel qui l'entoure, de devenir autonome; c'est-à-dire capable de se situer dans son environnement et complémentairement de l'influencer; c'est, en effet, en comprenant le jeu relatif de l'évolution de la société et de la sienne. propre qu'on est vraiment en mesure d'être un agent de changement.

A côté de cette définition, disons que l'éducation doit tenir compte (nous verrons comment):

a) des exigences de la formation professionnelle et d'ailleurs de l'évolution (ou dynamique) de ces exigences; b) des besoins culturels de la société; c) de la nécessité où l'on se trouve toujours de sélectionner une certaine forme d'élite.

L'école, prise dans le sens du système éducatif global, devra être simultanément le moyen et le lieu pour tous d'accéder à la compréhension critique de la culture et de la vie sociale, donc de pouvoir la discuter; et d'apprendre des métiers, ce qui signifie des savoirs et des savoir-faire permettant de choisir parmi des éventails et des gammes de métiers.

L'école doit, ce faisant, s'efforcer de former des citoyens responsables et non des sujets passifs. Le temps de présence à l'école doit constituer pour l'élève un certain apprentissage de la vie sociale, les élèves doivent pouvoir acquérir ou découvrir un savoir-être, à travers les rôles qu'ils adopteront tout au long de leurs études et les responsabilités qu'ils prendront dans le déroulement de celles-ci.

Enfin, l'éducation doit aussi être celle de la sensibilité et de la sociabilité.

L'éducation sera conçue comme un ensemble d'activités destinées à permettre à chacun:

- de conserver les connaíssances acquises en les reliant à sa vie affective concrète;

- de les accroître par une bonne information;

- de satisfaire les attentes de promotion, synthèse des mondes professionnel, culturel, civique et social;

- de permettre le changement sur ces divers plans;

- enfin, de développer la personne dans ses relations familiales et sociales.

\section{Education et emplol}

Etant donnée l'importance de ce problème, il nous semble utile de préciser notre pensée. 
Certes, il est inacceptable que le système éducatif \& produise > des élèves (jeunes ou adultes), en totale indifférence et en totale ignorance des besoins en main-d'œuvre. Mais il serait terriblement dangereux de céder à cette tentation si fréquente (même si elle n'est pas souvent avouée), d'adapter l'éducation à l'emploi et, ce, pour les raisons suivantes:

- les prévisions de l'emploi sont impossibles, sinon avec des erreurs très grossières, et d'autant plus grossières qu'on les fait au niveau regional; or, c'est au niveau régional qu'elles pourraient présenter de l'intérêt;

- contrairement à ce qu'on pense souvent, les s besoins $>$ en connaissances et aptitudes, correspondant aux différents emplois, ne sont guère plus prévisibles et sont, nous l'avons vu à propos des infirmières, d'abord définis en fonction de choix « politiques », mais de toutes façons, même lorsque les choix politiques ont été explicités, très difficiles à définir;

- l'adaptation emploi-éducation signifierait la négation de toute promotion et de toute.évolution, donc de toute possibilité de reconversion;

- l'éducation a bien d'autres objectifs que la formation professionnelle.

Dès lors, nous ne pouvons admettre que l'éducation doive être soumise à l'emploi et nous étudierons ultérieurement quelles solutions doivent alors être recherchées.

Nous verrons, en particulier, que les solutions doivent être trouvées, d'une part, dans les structures éducatives, mais, d'autre part, à l'extérieur: à ce sujet, disons que des efforts considérables doivent être faits, mais qui dépassent très largement l'Éducation nationale, pour valoriser certaines fonctions et, par exemple, celles de techniciens.

\section{POUR QUELLES RAISONS UNE EDUCATION PERMANENTE?}

Nous ne reprendrons pas ici toutes les raisons qui militent en faveur d'une éducation permanente. Ces raisons peuvent cependant se résumer en deux points:

\section{On ne peut pas tout faire à l'école}

On ne peut pas tout faire à l'école et ce, à la fois parce qu'il $y$ aurait trop à faire (d'où l'intérêt d'une éducation qui se continue et qui permet ainsi de réduire les connaissances à acquérir à l'école) et parce que certaines connaissances ou aptitudes ne peuvent y être acquises, parce qu'exigeant soịt des moyens qui ne peuvent s'y trouver, soit un stade de développement ou d'expérience que seuls des \& plus âgés \$ peuvent avoir.

\section{La compréhension d'un environnement constamment changeant exige une éducation permanente}

Accéder à la compréhension critique de la culture, dans la mesure où ce qui la constitue évolue constamment, ne peut se faire une fois pour toutes. Ii en est de même d'ailleurs du savoir et du savoirfaire; de même encore, bien sûr, de la formation de l'homme en tant que citoyen responsable.

L'éducation permanente est un moyen ou, mieux, un ensemble de moyens et non une fin en soi, moyens auxquels l'homme fait appel aux divers âges de sa vie, aux divers stades de son développement, aux diverses fonctions privées ou publiques qu'il occupe dans la société.

\section{UN PRINCIPE ESSENTIEL : LA CONTINUITÉ}

Tout ce qui précède suppose un changement profond des contenus, des méthodes et des structures, mais suppose d'abord admis un principe essentiel: la continuité entre l'éducation des jeunes et l'éducation des adultes, une continuité véritable et perçue par tous; d'autre part, tout adulte devra pouvoir de fait reprendre, à n'importe quel moment, son éducation, là où il l'avait laissée, mais il faut, d'autre part, que les adultes en soient parfaitement informés et réellement convaincus.

Les jeunes \&quitteront (d'ailleurs provisoirement) d'autant plus facilement le « train éducatif , (qu'ils n'encombreront alors peut-être pas) qu'ils sauront qu'ils pourront, sous de multiples formes, le reprendre et, pour avoir éventuellement perdu dans telle branche theorique parce que l'ayant abandonnée, en y gagnant sur telle autre en valorisant leur expérience professionnelle. A la limite, on devrait plutôt dire qu'on n'arrête pas ses études, mais qu'on en modifie le cours, c'est-d-dire le rythme, litinéraire et le mode de poursuite. Nous reviendrons sur ce point pour en voir quelques modalités pratiques: 


\section{LES RO̊LES DE L'ÉCOLE DANS LE SYSTEME NOUVEAU}

En dehors des rôles bien connus de transmission de la culture et d'apprentissage des métiers, à partir desquels se fait la formation à certaines aptitudes, l'éducation scolaire devra amener chaque jeune à une prise progressive d'autonomie, notamment vis-à-vis du savoir. Elle devra par conséquent, en outre, apprendre à utiliser les moyens et outils que l'on trouvera soit par la suite dans la vie « postscolaire », soit pendant la vie scolaire, mais en dehors de l'école: l'école devra en quelque sorte préparer les futurs adultes d vouloir et d pouvoir continuer à se former.

Mais, inversemènt ou comme complément, il faut souligner que certains éléments de la culture ne pourront pas être acquis après ou en dehors de l'école, si l'école n'y participe pas et ne donne pas ce qu'on pourrait appeler certaines « clés », n’ouvre pas certaines \& portes »: nous faisons allusion à la culture artistique, à la culture technique et à la formation civique et sociale.

Ainsi, l'école doit assurer quatre rôles:

1) apprendre des métiers; 2) apporter une formation de base; 3 ) apprendre à \& profiter de l'extérieur \$ (école parallèle); 4) mettre le futur adulte en état de pouvoir et vouloir continuer à se former au delà de sa vie scolaire et à poursuivre son développement et son épanouissement.

\section{COMMENT REMPLIR CES RÔLES ?}

Nous étudierons successivement les points 3 et 4, puis simultanément les points 1 et 2 , en nous posant chaque fois le problème des conséquences quant aux structures, aux contenus et aux méthodes.

\section{Apprendre aux jeunes à se former par des moyens extérieurs à l'école}

Les moyens de communication de masse s'étant à ce point intensifiés qu'ils bouleversent toute la culture de la société et, en tout cas, toute l'éducation, la jeunesse se trouve de plus en plus informée en dehors de l'école et n'est pas éduquée, sinon empiriquement, à se servir de cette information, à la critiquer, à la sélectionner.

Dès lors, un immense effort est à faire à l'école pour apprendre aux élèves à se servir des moyens qui existent à l'extérieur.
C'est ainsi que, dès l'école primaire, la formation par l'image et la formation à la lecture doivent être très développées. D'où conséquences sur:

\section{a) Les structures}

L'enseignement devrait se faire par périodes alternées, périodes où le maître explique et apporte des connaissances, périodes où il apprend à discuter, critiquer, sélectionner, comprendre l'information, et périodes où les élèves apprendraient à travailler seuls (ou en groupes, mais sans enseignant) ${ }^{3}$. De conférencier qu'il est actuellement, le maître passerait de plus en plus à un rôle d'assistance technique.

Sachant utiliser les outils et l'information, l'élève pourra de plus en plus travailler seul, non seulement pour réviser ou approfondir un enseignement suivi dans l'institution, mais pour entrer, de lui-même, dans des sujets qui lui auront été signalés comme utiles et qui ne feront l'objet d'aucun cours.

\section{b) Les méthodes}

Actuellement, la formation utilise essentiellement:

- le cours, l'élève ayant à écouter (et d'ailleurs, par là même à « choisir », ce qui ne lui est que peu enseigné, pas plus d'ailleurs que ne lui est vraiment enseigné la façon d'avoir à prendre des notes et à rédiger ce qu'il a entendu);

- des travaux pratiques, l'élève ayant à travailler dans un sens généralement très standardisé.

Il lui faudrait apprendre en plus, et cela ferait l'objet d'apprentissages spéciaux en classe, à:

- lire, c'est-à-dire comprendre la pensée écrite d'un auteur et la « pénétrer », c'est-à-dire la \& traduire » lui-même pour lui-même ${ }^{4}$;

- regarder l'image, et en particulier prendre du recul par rapport à elle, car celle-ci est souvent redoutable parce que captivante (et l'on est alors captif);

- et, à partir des informations, choisir et se situer, critiquer et sélectionner.

3. Cela est d'autant plus important que, de plus en plus, les enfants, en rentrant chez eux, sont soit trop seuls, soit - trop peu seuls * et qu'alors il devient indispensable que les classes leur soient ouvertes le soir et qu'ils puissent y travailler.

4. Nous voulons insister ici sur l'importance que revêt l'apprentissage de l'exploitation d'une lecture (qui est peutétre trop développé, parce qu'éventuellement desséchant, en littérature et pas du tout assez dans tous les autres enseignements). Peut-être apprendront-ils, en même temps, à aimer lire? 
La période scolaire deviendra beaucoup plus active, en même temps d'ailleurs que plus démocratique, par le droit à la participation personnelle et la responsabilité de chacun à sa propre formation.

\section{Former les jeunes de telle sorte qu'une fois adultes, ils souhaitent se former et puissent poursuivre leurs études}

Le projet que nous venons de développer est déjà de nature à aider le futur adulte à se former, car, dans la mesure où il aura été préparé à utiliser tous les moyens extérieurs, ces moyens n'étant pas différents lorsqu'il sera adulte, il sera tout naturellement poussé et prêt à les utiliser. Mais, d'autres solutions sont nécessaires qui, d'une part, mettront l'adulte en situation de vouloir poursuivre et, d'autre part, de pouvoir le faire.

\section{Le problème du « vouloir »}

Ce problème ne peut, bien entendu, se résumer en un seul terme, et nous ne voudrions pas réduire l'importance capitale des difficultés objectives et réelles qu'ont les adultes pour se former de manière continue. Mais, dans la mesure où le jeune, en quittant l'école, est, sinon décidé à ne plus se former, en tout cas très peu convaincu de l'intérêt qu'il y trouverait, il y a très peu de chance qu'il dépasse ses difficultés et celles-ci seront même accrues.

Ce que peut et doit apporter l'école nous semble d'une importance capitale: montrer au jeune que sa formation lui est \& utile », c'est-à-dire lui permet de répondre aux situations quotidiennes; il faut, pour y parvenir, que, tout au long de sa formation durant la période scolaire, le jeune apprenne à relier ses connaissances à son vécu ${ }^{5}$.

La formation des adultes est, pour le moment, gravement handicapée parce que ceux-ci ne sont pas en mesure d'établir un rapport réfléchi et critique entre leurs expériences personnelles ou professionnelles concrètes et les informations nouvelles qu'ils peuvent recevoir et qui sont nécessairement plus ou moins abstraites. Et nous ferons l'hypothèse que c'est parce que l'éducation des jeunes ne tient pas assez compte de ce problème que tout ce qui est nouveau pour les adultes, tout ce qui est connaissance transmise par les autres, est automatiquement classé dans

5. Faisons ici une distinction entre * son vécu * et celui du maître: lorsque le maître fait, en effet, appel au concret, il se réfère souvent plus au sien qu'à celui des élèves. le domaine de la théorie. C'est parce que les jeunes ne sont pas systématiquement habitués à établir des relations entre lois et exemples, entre règles et cas d'espèce, entre théorie et pratique que l'individu, une fois devenu adulte, ne pouvant plus trouver de relations entre ce qu'il fait et ce qu'il a appris, ne peut plus valoriser ses connaissances et, de ce fait, les perd peu d peu.

Comment, sinon, expliquer le fait que très nombreux sont les adultes de 25 ans qui ne savent déjà plus lire, dans le sens qu'ils ne comprennent pas ce qu'ils lisent (ils ignorent le sens de la virgule ou du point, le sens des mots relationnels, tels que \& car, mais, donc... » et qu'ils ne savent plus \& compter », en ce sens qu'ils ne savent plus la relation entre addition et multiplication? N'est-ce pas aussi pour les mêmes raisons que l'homme devient routinier et perd toute faculté de réflexion, voire la dénigre, et, prenant conscience de ce que la pratique se périme et de ce que la théorie est inutile, perd et le gout de la théorie et le goût de la pratique ${ }^{6}$ ?

(En fait, ce point a été souligné à maintes reprises dans la pédagogie des adultes et est considéré comme une évidence. Pourquoi ne pas en tenir compte dès l'éducation des enfants, comme si le vécu affectif n'existait pas pour eux, comme si l'on pouvait faire brusquement appel chez l'homme devenu adulte à cette part de lui-même, alors qu'elle n'avait jamais été valorisée dans son enfance et son adolescence? C'est peut-être ce qui donne ce sentiment de merveilleuse révélation, proche aussi de la libération, à un adulte qui, pour la première fois depuis sa scolarité, fait l'épreuve de pouvoir réapprendre avec succès.)

Il faut donc lier vécu et formation. Mais comment? Cela suppose un certain nombre de réformes qui portent sur:

\section{a) Les structures}

Du temps doit être laissé pour « accroître le vécu », soit par des visites, soit par des travaux en commun.

\section{b) Les contenus}

Tous les contenus ne se prêtant pas autant au lien entre éducation et vécu et tous les enfants n'y étant pas sensibles de la même manière, le choix des contenus devra être diversifié de façon à en tenir compte. En particulier, une raison de non-démocratisation de l'enseignement est incontestablement que certains contenus, considérés comme fondamentaux et qui cons-

6. Voir à ce sujet le document de M. Tietgens, Conseil de l'Europe. 
tituent la partie principale de l'éducation, sont trop éloignés du vécu de certains enfants (n'est-ce pas le cas de la littérature classique pour les fils d'agriculteurs et d'ouvriers ?). La démarche et le cheminement devront être alors différenciés selon les enfants.

\section{c) Les méthodes}

Le problème est de savoir comment utiliser le vécu des enfants et comment le connaître.

Ce vécu peut en fait apparaître, se manifester, donc s'exploiter, de deux manières:

- Par les questions que les enfants posent: or, bien souvent, non seulement, on ne les écoute pas ou on ne les entend pas, et l'on n'y répond donc pas (soit qu'on ne veuille pas, soit qu'on ne le puisse pas), mais surtout on leur en pose d'autres dont ils ne saisissent, ni le sens, ni la raison. Cela est d'autant plus grave (et d'autant plus évitable) qu'il s'agit de contenus « vivants », tels que sciences physiques, sciences naturelles, ou géographie. Les questions posées par le maître sont très souvent du type: On donne ceci, on vous demande de trouver cela. $\gg$ Or, dans la vie, non seulement on ne donne rien, mais encore on ne demande rien ১. Au contraire, il faut, dans la situation dans laquelle on se trouve, se poser des questions, mais qui ne sont pas données. La créativité et l'imagination passent par l'apprentissage d̀ se poser des problèmes, d se trouver les données et les inconnues, beaucoup plus finalement qu'd résoudre des problèmes posés par d'autres.

- Par l'analyse des représentations qu'ont les enfants des phénomènes qui les entourent. Par e représentations $\triangleright$, il faut entendre les images qu'ils se font et qui sont plus ou moins différentes de la réalité (par exemple, une image qu'on rencontre très fréquemment est que la pesanteur est « due à l'atmosphère qui pousse sur les objets $\gg$ ).

Or, ne pas tenir compte, dans la formation, de ces représentations a très souvent comme effet (l'expérience le prouve) que, non seulement ces représentations sont conservées après l'enseignement, mais sont même renforcées, inconsciemment bien sûr, l'élève sélectionnant dans ce qu'il entend ce qui va dans le sens de sès représentations. Disons qu'en tout cas l'existence des représentations freine l'apprentissage, alors que la discussion ouverte et systématique de ces représentations est, au contraire, un excellent point de depart pour la conceptualisation et l'assimilation des connaissances. « Développer \$ ne peut se faire qu'en «partant de ». Plus on se relie à la représentation qui est une forme intellectuelle du vécu, plus il y a de chances pour qu'on développe la personne.

La culture est en quelque sorte un système structuré, organisé, qui comprend des parties qui ne sont pas indépendantes les unes des autres. Cette entière dépendance se manifestera ainsi: tout fait nouveau est immédiatement assimilé ou, au contraire, rejeté ou modifié avant d'être assimilé; et, s'il entre en conflit avec les éléments les plus fondamentaux de cette organisation, c'est éventuellement lui qui désintègre la culture.

\section{Former les jeunes de telle sorte qu'une fols adultes lls solent capables de continuer leur éducation après leur sortie de l'école}

C'est un problème de méthodes, mais c'est aussi un problème de contenus.

\section{Le problème des contenus}

Actuellement, la formation de base n'est constituée que d'humanisme ou de sciences. Plus exactement:

- pour certains, elle ne comprend effectivement (jusqu'au baccalauréat) aucune autre formation, alors que, pour d'autres et malgré les efforts faits dans le sens d'une déspécialisation (remplacement des CAP par les BEP), elle est très tôt professionnelle.

Nous ne saurions trop insister sur la nécessité d'élárgir le champ de cette formation de base dans les trois directions de la culture artistique, de la formation technique et de la formation civique et sociale. Il s'agit donc d'ajouter des contenus à ceux qui existent déjà.

Mais quel temps restera alors pour les contenus qui sont aujourd'hui enseignés, quelle réduction doit leur être affectée, et ce d'autant plus qu'un temps considérable serait consacré à la formation méthodologique et à la formation à l'utilisation des moyens extérieurs?

En fait, deux facteurs permettront des réductions:

- parce qu'il y a éducation permanente, il est possible de remettre à plus tard un certain nombre de contenus que l'on enseignait normalement à l'école; 
- d'autre part, l'étude des contenus, effectués selon la méthode explicitée à propos de la définition des objectifs de l'enseignement, doit permettre un gain de temps appréciable, par la suppression pure et simple de certaines parties et l'apprentissage moins approfondi et moins encyclopédique d'autres?.

En tout état de cause, faute d'apporter une éducation artistique, technique, civique et sociale aux jeunes, leur formation extra et postscolaire a toutes chances d'en être profondément affectée.

\section{a) L'éducation artistique}

Sans vouloir traiter à fond du sujet, nous soulignons que le besoin culturel naît de l'éducation et nous insistons sur le fait que l'Éducation nationale doit prendre une part considérable au développement culturel.

L'école doit, par un effort massif, permettre à tous les enfants de voir quels sont leurs goûts et leurs possibilités d'épanouissement dans l'ensemble des activités artistiques. Cela signifie que tous les enfants devront recevoir une sensibilisation aux arts, et qu'en dehors de cette sensibilisation, chaque enfant devra pouvoir choisir, parmi toutes les formations artistiques - et au même titre que n'importe quelle autre option - celle dans laquelle il pourra s'épanouir.

Il n'est pas question ici de développer des méthodes: disons seulement qu'il n'existe pas qu'une seule méthode pour apprendre à aimer un art et s'y éduquer et que la technique de formation artistique ne doit surtout pas cacher et précéder la sensibilisation: alors qu'en France, de nombreuses personnes sont considérées comme très peu douées en dessin ou en musique, le Japon, par exemple, est arrivé, par la diversité de ses méthodes et les efforts qu'il y déploie, à ce que la quasi-totalité de ses enfants dessinent bien et aiment le dessin et jouissent profondément de la musique et du théâtre. Et comment ne pas être frappé par la passion que manifestent les jeunes Japonais pour la lecture, au point que toutes les librairies sont remplies de jeunes qui, debout, lisent gratuitement pendant des heures ?

\section{b) L'éducation technique}

Nous avons déjà insisté sur l'importance capitale qu'il y avait à relier la théorie à la pratique: il nous

7. N'est-ce pas un affreux crime de clèse-culture s que d'admettre que de nombreuses parties pourront être supprimées, tout simplement parce qu'elles ne laissaient finalement aucune trace? paraît essentiel que tous les enfants reçoivent; au cours de leur existence scolaire, une éducation technique prise dans le sens de relier le travail manuel, l'expérience et l'abstraction.

\section{Cela évitera:}

- que de nombreux enfants, qui n'ont fait que des études scientifiques ou littéraires, n'aient aucune idée de ce qu'est la technique et, la méconnaissant, la méprisent;

- que d'autres, parce qu'ils sont considérés comme peu doués dans les études humanistes, soient rejetés vers le technique, au lieu, pour certains, de le choisir par goût, et pour d'autres de chercher d'autres orientations qui leur plairaient davantage.

Une formation technique étendue à tous permettrait, au lieu d'une orientation par l'échec, une orientation davantage déterminée en fonction des goûts et des possibilités de chacun.

\section{c) L'éducation civique et sociale}

Elle serait basée sur l'étude de certains problèmes essentiels, mais devrait surtout s'appuyer sur un apprentissage de la responsabilité.

\section{Quelles méthodes adopter pour apprendre à apprendre?}

Ce problème exigerait à lui seul de tels développements que nous ne pouvons insister. Soulignons seulement les points qui semblent les plus essentiels:

- A chaque objectif correspondent une ou plusieurs méthodes, et c'est une raison supplémentaire pour définir ces objectifs. Par exemple, pour informer, l'audio-visuel et la conférence sont particulièrement adaptés. Pour développer un «moyen d'expression \ (pris au sens où nous l'avons défini précédemment), l'exposé suivi de discussions de groupe est une bonne méthode. Seule la répétition et un travail personnel apportent l'outil et la formation à la méthodologie passe par la critique de la méthode elle-même.

- Il faut abolir le tabou selon lequel, à l'école, on ne parle pas de l'école. Il faut montrer aux élèves comment remettre constamment en cause l'apprentissage lui-même, comment se démonte leur propre apprentissage. 
- Enfin, l'évaluation des résultats n'est plus l'apanage du maître et doit être faite en commun avec l'élève. Tout doit être mis en cuvre pour remplacer l'évaluation par une \& auto-évaluation \$ prise dans le sens de « donner à l'élève la possibilité de se situer lui-même en permanence et d'analyser ses propres erreurs $\gg$; cela ne signifie évidemment pas que l'élève se met des notes, ni non plus que le professeur s'abstient de dire à l'élève ce qu'il pense, mais que professeur et élève étudient ensemble les difficultés et les insuffisances de l'élève et les moyens à mettre en ouvre pour progresser. L'auto-évaluation devient, dans ces conditions, un acte pédagogique essentiel et ne peut prendre un sens que si élève et professeur ont bien précisé et intégré les objectifs à atteindre en commun.

A noter que l'attestation de capacité, à l'issue d'une période éducative, reste évidemment le fait du maître, mais, en travaillant sur des groupes en nombre restreint (25 au maximum, 15 si possible), les examens peuvent être remplacés par un véritable contrôle continu, et, en cas de « désaccord » entre l'élève et le professeur, l'élève pourra faire « appel », c'est-à-dire passer un examen chez un autre professeur.

\section{Apprendre des métiers et apporter une formation de base}

$\mathrm{Si}$ nous traitons ensemble ces deux problèmes, c'est précisément qu'il nous semble qu'ils ne sont pas dissociables s'ils sont réunis sous le signe éducation s. Cela veut dire que le conditionnement ou le développement sont beaucoup plus fonction de la méthode que du contenu: un contenu \&professionnel > peut être sujet de développement de la personne, alors qu'à la limite un contenu artistique peut être conditionnant.

En dehors des questions de méthodes,

\section{a) c'est la structure qui est ici déterminante}

Nous ferons les hypothèses suivantes:

- A partir de l'âge de 14 ans, les élèves doivent pouvoir par étapes successives de 2 à 3 ans, obtenir des diplômes et des grades.

- Le diplôme ouvre la porte des professions, le grade est une reconnaissance d'un niveau. Le diplôme est donc la somme d'un grade et d'une formation professionnelle.
- Tout élève ou adulte titulaire d'un diplôme ou du grade de même niveau peut, à tout instant ${ }^{8}$, reprendre ses études, soit pour préparer un diplôme de même niveau, soit pour élever son niveau et obtenir un grade supérieur.

- Il doit exister, à chaque niveau, de nombreux diplômes très diversifiés.

- Pour passer d'un grade au grade supérieur, il faut avoir fait la preuve de sa capacité dans un certain nombre d'unités. La formation professionnelle est elle-même décomposée en unités. Les unités sont capitalisables, l'ordre dans lequel elles sont suivies, sans être quelconque, doit être très souple pour permettre aux jeunes de suivre le cheminement qui correspond le mieux, à tout instant, à leurs motivations, à leurs capacités et à leur « vécu 》.

- Le tronc commun entre tous les diplômes et, ensuite, entre les familles de diplômes, doit constituer la part principale de l'éducation. Il doit y avoir un véritable continuum de la base au sommet, à partir duquel se détacheraient les branches professionnelles.

- Chaque stade comporterait une partie obligatoire du tronc commun, une série d'options et au moins une activité d'épanouissement.

Tout le système éducatif fait ainsi l'objet de division en unités, chaque diplôme correspondant à un groupe d'unités.

Ce système devrait permettre aux élèves, dès après 14 ou 15 ans, de se décider à tout instant, à tout niveau, à quitter le tronc commun pour acquérir tel diplôme, donc obtenir une sanction de leurs efforts et de quitter l'école (quand ils le voudraient, après l'âge légal), pour y rentrer à n'importe quel autre moment, en retrouvant le « train à la gare » où ils en seraient sortis.

Mais on peut aller encore plus loin dans la réforme des structures en rompant l'unité de temps l'année - et l'unité classe $\gg$, pour les remplacer par un système d'unités de contenu suivies par des groupes ayant leurs rythmes autonomes de progression.

Il faut entendre, par là, le fait que, au lieu que, comme actuellement, tous les enfants d'une même tranche d'âge se trouvent dans une même classe, pour apprendre, à la même vitesse, toutes les matières et ne peuvent passer d'une classe à l'autre que lors-

8. Eventuellement après une courte actualisation. 
qu'ils ont satisfait à l'ensemble des matières, à l'inverse les enfants sont, pour chaque matière, répartis en groupes, tel élève pouvant parfaitement être « en $3^{\circ}$ > en mathématiques et en $5^{\circ}$ > dans sa langue maternelle ${ }^{9}$. Les groupes sont donc autonomes et ont leur rythme propre (en pratique, il y aurait deux ou trois vitesses). Ces groupes seraient d'ailleurs autonomes, non seulement dans leur rythme, mais dans le choix de leurs méthodes.

\section{b) Que sont alors les contenus, les unités?}

Dans le paragraphe sur les objectifs, nous avions été amenés à proposer plusieurs catégories d'objectifs, à savoir: les objectifs d'information, d'expression, d'outil ou de méthodologie.

Reprenant cette classification, nous dirons que certaines unités seront à dominante d'information, d'autres à dominante « d'expression », d'autres d'outil et d'autres enfin de méthodologie.

Les unités constituant les « grades » porteront, soit sur des contenus de base, soit sur des contenus techniques. Les unités, qu'il faudra suivre en sus de celles du grade pour obtenir un diplôme, seront « professionnelles $\gg$.

Certaines unités de base ou de techniques seront communes à tous les diplômes, d'autres à des familles de diplômes et d'autres enfin seraient totalement c libres \$.

Donnons trois exemples pour illustrer ce qui vient d'être dit:

- Il n'y aurait entre la préparation pour le professorat de sciences naturelles et celle d'un biologiste que très peu d'unités différentes; les quelques unités supplémentaires, celles qui conféreraient le diplôme de professeur de sciences naturelles, consisteraient à apprendre comment enseigner les sciences naturelles.

- Deux étudiants, qui auraient fait des études de chaleur, thermodynamique, électricité, mathématiques et chimie, pourraient, à ce moment, diverger pour devenir:

- le premier, ingénieur thermicien: il suivrait alors une unité de thermique industrielle, une unité de sociologie, une unité d'économie et une unité de résistance des matériaux,

9. Voir à ce sujet le document du Recteur Janne, Conseil de l'Europe.
- le deuxième, professeur de physique: il suivrait une unité de pédagogie et des unités de physique (unités qu'il n'aurait pas encore suivies, mais qui ne seraient plus traitées en unités méthodologiques mais en unités « professionnelles », c'est-à-dire sous une forme plus rapide et moins approfondie).

- Dans la préparation actuelle du diplôme de BEP d'électromécanique, il y a des contenus du type dessin, mesures électriques, technologies qui sont absolument spécifiques à ce BEP. L'idée est, au contraire, de faire de ces contenus des unités méthodologiques en général (unités qui emprunteraient leurs exemples d'ailleurs à la fois à l'électricité et à d'autres branches), et c'est dans les unités de formation professionnelle qu'in fine les élèves apprendraient ce qu'il y a de spécifique à la profession à laquelle ils se préparent.

Les contenus de la formation de base seraient finalement triplement modifiés:

1) Trois contenus seraient donc ajoutés (art, technique, civique);

2) Chaque contenu est repris à la lumière de la définition des objectifs des unités;

3) Le fait qu'il y ait continuité depuis le primaire jusqu'au supérieur doit faire repenser l'ensemble et la répartition de ces contenus.

Notons encore quelques points:

- Il est important de ne pas multiplier les unités dans une même année, ceci pour augmenter la concentration des efforts.

- A partir d'un certain âge, il paraît préférable de travailler le même contenu pendant deux ou trois heures de suite (ce qui ne signifie ni qu'il n'y a pas de pause, ni surtout que l'objectif et la méthode pédagogique resteront les mêmes pendant tout ce temps).

- Les unités professionnelles gagneront en efficacité, si l'on y fait participer de récents titulaires du diplôme correspondant.

- D'une manière plus générale, il peut être extrêmement intéressant d'ouvrir les classes aux parents (sous réserve qu'ils n'aillent pas dans la classe de leur enfant). C'est un remarquable moyen de développer l'éducation permanente. 


\section{c) Etude critique}

Par sa souplesse et par la prise de responsabilité effective qu'il donne aux élèves dans leur formation, un tel système devrait permettre à chacun de savoir, à tout moment, où il en est de sa progression et de l'assumer.

Il contribuerait, d'autre part, en grande partie, a résoudre les problèmes de l'emploi et de la sélection. En effet, si, d'une part, à tout instant, les élèves, les étudiants, les adultes sont informés des besoins en emplois tels qu'ils sont estimés et si, par ailleurs, ils peuvent, à tout moment, changer facilement de métier, en suivant telle ou telle unité spécialisée supplémentaire, les risques d'inadéquation entre besoins et ressources en main-d'œuvre seront très limités; par ailleurs, la sélection recevra une solution dans la mesure où les êlèves, les étudiants, les adultes sauront à tout instant qu'ils pourront, de fait, partir, revenir, changer et reprendre. Alors, nous l'avons vu, on peut faire l'hypothèse qu'ils quitteront l'Université avec moins de crainte, car on quitte d'autant plus facilement l'école que l'on sait que l'on peut y revenir et que, surtout, l'on sait que, pour avoir quitté l'école à tel stade, ce n'est pas comme le recalé de quelque chose s, mais à l'inverse, comme le futur reçu de..., le "prêt à devenir quelque chose ».

Mais, bien entendu, il peut être fait à ce système de nombreuses critiques, l'important étant d'ailleurs de les connaître afin de les traiter.

Citons-en quelques-unes:

- Grande complexité pour organiser des quantités de petites unités. En fait, il faut retenir que, plus il y a d'élèves ou d'étudiants, et moins il est difficile de résoudre ce problème; seul un enseignement de masse permet l'individualisation (dans les institutions à faible nombre d'élèves, peut-être est-ce impossible et faudra-t-il réduire nos ambitions?).

- On peut craindre qu'il y ait une sélection antidémocratique dans la mesure où des groupes lents comporteraient surtout des étudiants qui, par suite de leurs conditions de vie, auraient été moins bien préparés, d'où un drainage social au départ. Précisément, c'est par des méthodes pédagógiques différenciées que l'on peut et doit arriver à ce que des étudiants ne \& restent \& pas lents. D'autre part, on peut penser qu'il n'y a aucune traison pour que des étudiants soient lents partout et qu'au contraire uls seront lents dans certaines matières et rapides dans d'autres.

- On peut craindre que la formation donnée ainsi, au sein de petits groupes autonomes, amène à une absence de vue générale et à un morcellement de la culture. Si l'on veut réduire ce danger, il faudra, au lieu de travailler sur des matières, travailler sur des groupes de matières.

\section{IMPLICATIONS DE CES HYPOTHESES}

\section{Rôle de l'information et de l'orientation}

Il est évident que l'information et l'orientation jouent un rôle capital: l'information, pour permettre à tout étudiant, qu'il soit jeune ou adulte, de savoir où en est la situation de l'emploi; l'orientation, qui devient alors un acte pédagogique constant, pour permettre à chacun de se situer par rapport aux autres et par rapport à sa propre progression.

\section{Rôle et formation des maîtres}

Le rôle des maîtres est donc un rôle entièrement nouveau et leur formation prend une importance dont il est difficile, tant elle est grande, d'apprécier la valeur. Leur vie propre en sera totalement modifiée, ne serait-ce que par l'influence qu'ils devront prendre dans leur collectivité.

\section{Locaux}

Il est clair que la conception des locaux doit être entièrement repensée.

\section{COMMENT COMMENCER TOUT CELA?}

Si de telles hypothèses n'étaient pas, a priori, rejetées en bloc, elles devraient alors faire l'objet de réflexions longues et approfondies avant même d'être diffusées, car, pour exaltantes 'qu'elles soient, certaines d'entre elles sont probablement utopiques.

Trois étapes devraient alors être suivies.

\section{Étape de réflexion, qui durerait une année}

Des groupes de réflexion devraient être mis en place pour étudier:

a) Les contenus, à la fois de manière horizontale, c'est-à-dire à l'intérieur des « matières », pour voir 
comment les concevoir de façon nouvelle en suivant ou en modifiant nos hypothèses sur les objectifs, et de manière verticale, c'est-à-dire pour voir comment construire le e continuum s depuis l'école primaire jusqu'à l'Université. Notons à ce sujet qu'il faut absolument éviter que le primaire, le secondaire, le technique et le supérieur ne travaillent chacun de leur côté. C'est en cela que les recherches devront être verticales (précisons à ce sujet que le primaire a encore trop souvent comme finalité le CEP et forme encore trop un système clos).

b) Les structures des unités, des diplômes, des classes.

c) Les méthodes, tant pour apprendre les contenus que pour se servir des moyens extérieurs.

\section{Étape d'expérimentation quil devra durer plusieurs années}

Il s'agira d'expérimenter ce qui aura été proposé au cours de la première étape.
Notons que cette étape passe par:

- la formation des maîtres dans la ligne des réformes prévues, alors qu'en toute rigueur il n'y en ait point qui y ait été préparé;

- la réorganisation des structures des institutions pour qu'elles cessent de faire obstacle au nouveau système d'éducation que l'on chercherait à implanter;

- la réflexion sur l'amont et l'aval de chaque élément de la réforme projetée.

\section{Etape de systématisation}

Rien ne peut être dit à ce sujet, sinon:

- qu'il est probable que bien des principes se trouveront modifiés après expérience et qu'il faut surtout que se mette en place une structure qui engendre sa propre remise en cause. 